\title{
A New Digital Audio Watermarking Algorithm
}

\author{
Ping Ren ${ }^{1,2, *}$ \\ ${ }^{1}$ Suzhou Vocational University, Department of Computer Engineering, Suzhou 215104, Jiangsu, China \\ ${ }^{2}$ Jiangsu Province Support Software Engineering R\&D Center for Modern Information Technology Application in \\ Enterprise, Suzhou 215104, Jiangsu, China
}

\begin{abstract}
In this paper a new digital audio watermarking algorithm is proposed based on B-spline approximation. The algorithm takes the advantage of non-uniform B-spline wavelet, namely, the number of low-frequency components can be arbitrarily selected, so, it is very flexible. Meanwhile, it avoids the complex calculations of non-uniform B-spline wavelets. The simulation experiments show that this algorithm guarantees the inaudibility of the watermark, and it is robust against common attacks as well.
\end{abstract}

Keywords: Digital audio watermarking, B-spline wavelet, inaudibility, robustness.

\section{INTRODUCTION}

With the rapid development of internet technology, a variety of digital multimedia information, such as text, image, audio, and video can easily spread via networks. At the same time, how to protect the copyrights of these digital products becomes an urgent problem. In recent years, as an effective way to protect copyright of digital products, digital watermarking technology has been paid great attention and has become a hot topic in the field of information security [1]. Among a lot of digital watermarking algorithms, the wavelet-based algorithms have been paid much attention for the reason that wavelets have the multi-resolution characteristic. However, the wavelets used in digital watermarking are binary wavelet, therefore, the data number of low frequency and high frequency are both halves of the number of original data after one-dimensional wavelet decomposition is carried out, so it has some limitation. Non-uniform B-spline wavelet overcomes such problem [2], but it is very complicated to construct and compute the wavelet basis, which limits its practical application in the digital watermark. Audio signal intensity as masking tone, weak energy and strong signal masking cannot be signal ear perceive be masking tone.

Watermark signal to modify the form of the original audio data embedding, how to effectively embed the watermark signal, not only affects the imperceptible watermarked, but also in resistance to a variety of audio assault on robustness, therefore, embedding algorithms become a focus of research. Audio watermarking technology is in the premise of not affecting the quality of the audio signal, a method for integrity checking to the audio signal to embed the watermark signal is used to protect the copyright or. The main application of robust audio watermarking is to protect the developer or the author's copyright. Audio watermarking technique including three parts extracted watermark generation, embedding of watermark. The watermark embedding process is the research focus of watermarking technology is difficult, the general method using superposition or quantization watermark information will be mixed into the original audio signal in time domain or the transform domain coefficients, the watermark is embedded into the host signal is difficult to remove. In order to promote the practical application of audio watermarking, usually hope the watermark detection can realize blind detection.

Masking tone in the former, masked tone in the situation called backward masking or forward masking, masked tone in the former, masking tone in the situation referred to as forward masking or backward masking, forward masking, masking is usually exist at the same time, backward masking generally disappeared in $50-200 \mathrm{~ms}$ masking tone, forward masking in general masking tone appeared before the $5 \sim 20 \mathrm{~ms}$ masking can hide more information. The main works in this article are as follows:

Firstly, audio data is divided into several segments. For each segment, each value is regarded as a control point and a B-spline curve is constructed. Secondly, the curves are approximated by the B-spline curves with less control points, the differences between the original curves and approximating curves are calculated and recorded [3]. After that, the watermarks are embedded into the control points of approximating curves. Finally, the watermarked audio data are obtained by summing watermarked approximating curves and difference curves (similarly to the wavelet reconstruction). To analyze the digital audio watermarking principle from the angle of signal processing, the process of embedding digital audio watermarking signal is seen as the superposition of weak signal in strong signal, according to the characteristics of human auditory system, as long as the guarantee that the added signal under the human auditory system threshold or in audio signals have a stronger voice can cover up to add 
sound, the human ear can not feel the signal changes, or feel very small changes. In order to achieve the purpose of embedding watermark will carry on the adjustment to the original audio signal, but considering the time and frequency characteristics of the audio signals, this adjustment is little impact on human hearing it $[4,5]$.

Digital audio watermarking technology has a very broad application prospects: on the one hand, can realize the protection and authentication of digital audio copyright audio watermarking technology, which is the application of watermark technology the most important. So digital watermarking can be used to solve the problem of equitable ownership; on the other hand, can be used for audio piracy tracking, its purpose is to transfer legal receiver information instead of the data source information, is mainly used for individual identification data release prints. Information security technology is the traditional cryptography technology to protect copyright and security based on.

This paper based on digital audio signal as the research object, research on the copyright protection of digital audio works, transform the study mainly focused on the digital audio, aims to propose watermarking information is embedded into the appropriate algorithm. In view of the deficiency of audio watermarking in transparency and blind detection, proposes an audio watermarking algorithm based on wavelet transform coefficient quantization, the algorithm has good transparency, in the ideal case can achieve zero watermarking, reduce the computation complex degrees, to achieve blind watermark detection system. Analysis of the advantages and disadvantages and summary of audio watermarking against synchronization attack, causes the audio watermarking against synchronization attack ability is limited, the existing synchronization between the watermark is unable in the robustness and transparency to effectively balance, presents a self synchronizing audio watermark algorithm based on DWT.

Masking effect can be further divided into temporal masking and simultaneous masking, temporal masking is refers to between the different time sound mutual masking, is by the human ear to time-resolved decision mechanism, simultaneous masking refers to the mutual masking at the same time between different frequencies of sound, shown in Figure 1 is the simultaneous masking, simultaneous masking is due the human ear to the voice of the determined frequency resolution mechanism.

\section{METHODOLOGIES}

\subsection{Basic Theory}

In order to describe the complex shapes and a local nature, B-spline curve equation can be written as:

$$
p(u)=\sum_{i=0}^{i=n} d_{i} N_{i, k}(u)
$$

Which, $\quad d_{i}(i=0,1, \cdots, n)$ is the control points, $N_{i, k}(u)(i=0,1, \cdots, n)$ is called the $\mathrm{k}$-th $\mathrm{B}$-spline basis func- tions, which is referred to as B-spline. B-spline recurrence is defined as:

$$
\begin{aligned}
& N_{i, 0}=\left\{\begin{array}{l}
1, \text { if } u_{i} \leq u \leq u_{i+1} \\
0, \text { other }
\end{array}\right. \\
& N_{i, k}(u)=\frac{u-u_{i}}{u_{i+k}-u_{i}} N_{i, k-1}(u)+\frac{u_{i+k+1}-u}{u_{i+k+1}-u_{i+1}} N_{i+1, k+1}(u) \\
& \operatorname{pr} \frac{0}{0}=0
\end{aligned}
$$

The recurrence formulas show that we want to determine $N_{i, k}(u)$, we need to use $u_{i}, u_{i+1}, \cdots, u_{i+k+1}$ nodes.

There are a couple of different ways to describe a Bspline curve. In its most basic form it looks just like the Bezier formulation.

$$
W^{m}(u)=\sum_{i=0}^{n} P_{i} B_{i, m}(u)
$$

where $\mathrm{P}$ is the control point vector, $\mathrm{B}$ the basis function, $\mathrm{n}$ the number of control points and $m$ the degree of the curve. Equation (5) is simple to understand, and uses the fact the B will automatically return 0 if the control point being evaluated is "out of reach" of $u$. This is however not very ecient since we for every u must evaluate the basis function for every $P_{i}$.

The main properties [6] of B-splines are as follows: (1) The B-splines of degree $n$ with a given knot sequence that do not vanish over some knot interval are linearly independent over this interval. (2) Each point is affected by $\mathrm{k}$ control points. (3) Each control point is affected $\mathrm{k}$ segments. (4) Uniform B-splines don't interpolate de Boor control points. (5) There are recursive, standard, local support and differentiability.

To analyze [7-10] digital audio watermarking from digital communication point of view, the process can be embedded in the digital audio watermarking as a narrowband signal (digital audio watermarking information) to a broadband signal through a certain algorithm (i.e., the original audio signal) such a frequency modulation process of embedded signal is usually dispersed, and hidden in the original audio signal. The watermark detection process is equivalent to the information demodulation process, mainly from the broadband signal in narrow-band $1 \mathrm{H}$ demodulation.

\subsection{Overall Design}

In this paper, the audio data is in a meaningful binary image embedded watermark, the basic idea is: First, audio data is divided into several sections with each data as the control point (B-spline function corresponding coefficient) to construct B-spline curve, and then contains fewer control points of B-spline curve to approximate the original curve (corresponding to the low frequency components in the wavelet decomposition). Calculate the approximate curve and the difference between the original curves (corresponding to the 


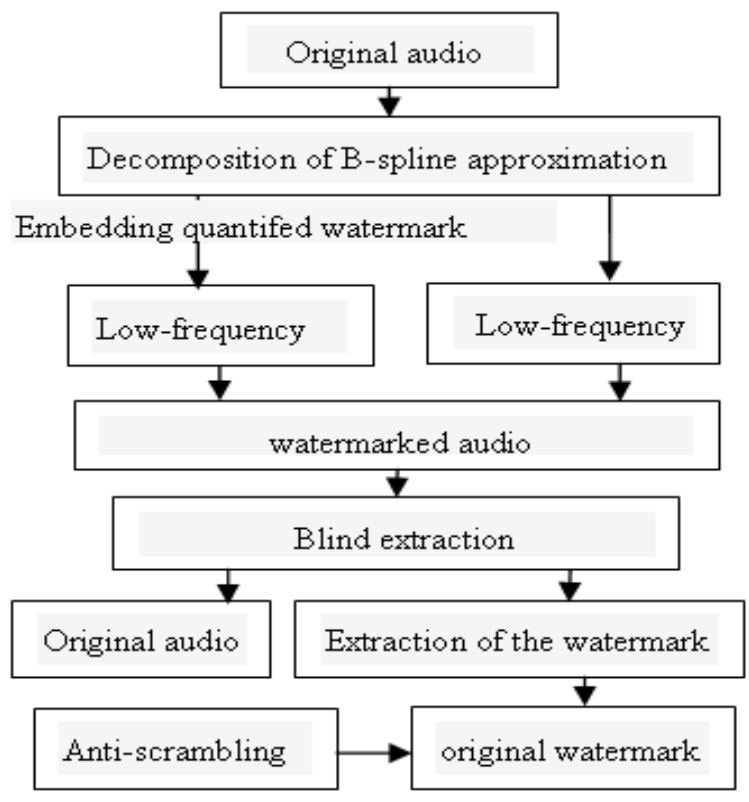

Fig. (1). Embedding and extracting watermarking of B-spline approvimation.

wavelet decomposition of the high frequency components). The watermark is embedded to approximate the curve control points, and then the sum and difference of curve (similar to the wavelet reconstruction) has been watermarked audio data. In addition, in order to increase the security of the embedded watermark, we first scramble the encrypted watermark information. Watermark extraction is the reverse process of watermark embedding. The watermark embedding and extracting process are shown in Fig. (1). Digital audio watermarking from digital communication point of view, the process can be embedded in the digital audio watermarking as a narrowband signal (digital audio watermarking information) to a broadband signal through a certain algorithm (i.e., the original audio signal) such a frequency modulation process of embedded signal is usually dispersed, and hidden in the original audio signal. The watermark detection process is equivalent to the information demodulation process, mainly from the broadband signal in narrow-band $1 \mathrm{H}$ demodulation [11-14].

\subsection{Watermark Scrambling}

At present audio watermarking and there is no uniform standard, standard perception evaluation, robustness, capacity. Perceptual evaluation to evaluate the effect of mixed audio on the human auditory system, used to evaluate the robustness of watermarking system robustness, capacity with many meter evaluation of watermarking embedding algorithm to embed the watermark information. Base in digital watermarking for the $\mathrm{W}=\{\mathrm{w}(\mathrm{i}, \mathrm{j}), 1 \leq \mathrm{i} \leq \mathrm{a}, 1 \leq \mathrm{j} \leq \mathrm{b}\}$, in which $w(i, j) \in(0,1)$ binary watermark image is on behalf of the $i$ row $j$ column pixel gray values. To enhance the security of the watermark and robustness, the watermark before embedding the image pre-processing, using the method of scrambling modulation of the watermark is encrypted to eliminate the watermark pixel spatial correlation. The algorithm uses Arnold image [4] scrambling technology. Arnold transform is:

$$
\left(\begin{array}{l}
x^{\prime} \\
y^{\prime}
\end{array}\right)=\left(\begin{array}{ll}
a & a b-1 \\
1 & b
\end{array}\right)\left(\begin{array}{l}
x \\
y
\end{array}\right) \operatorname{Mod}(N) a, b \in Z
$$

The transformation is one to one. The image can be used iteratively to produce different results images until the required data appears. Arnold transformation has periodicity, when the iteration to a step, it will regain the original image. The use of technology of watermark scrambling pretreatment can improve the security of the watermark information and enhance the ability of the watermark against malicious attacks.

\subsection{Watermark Embedded and Extracted}

First, the audio data and watermark data are divided into the same several sections and construct B-spline curve. Then proceed is as follows:

(1) Approximation function is obtained embedding the low-frequency components.

(2) Express B-spline curve and record high-frequency coefficients. Using quantitative methods embed in the low frequency part of the watermarked coefficients. The quantitative approach is: If the binary watermark pixel value is 0 , the low frequency data by the same location even after quantization or binary watermark pixel value are 1 . If the same location after the low-frequency data is quantified for the odd, the original audio low frequency data is unchanged. otherwise, we adjust the frequency by plus or minus 1 the amount of data, allowing for the watermarked frequency of each volume. If the corresponding position of the watermark value is 0 , then quantification is even. If the corresponding location watermark value is 1 , then quantified as odd.

(3) Get the watermarked audio data.

Watermark extraction steps are as follows:

- The watermark audio signal is segmented.

- The compositions of each audio watermark implement B-spline approximation decomposition to get low-frequency components.

- Use quantitative factors to quantify and determine the parity. Subsequently receive a one-dimensional watermark sequence.

- The watermark sequence is from 1-dimensional to two-dimensional images. Then scramble to get the anti-two-dimensional watermark.

- By calculating the normalized correlation (NC) to evaluate the extraction of the watermark information and the similarity between the original watermarks.

$$
N C\left(w, w^{\prime}\right)=\frac{\sum_{i=1}^{a} \sum_{j=1}^{b} w(i, j) w^{\prime}(i, j)}{\sqrt{\sum_{i=1}^{a} \sum_{j=1}^{b} w(i, j)^{2}} \sqrt{\sum_{i=1}^{a} \sum_{j=1}^{b} w^{\prime}(i, j)^{2}}}
$$

$w(i, j) \in(0,1), 1 \leq i \leq a, 1 \leq j \leq b$ is binary watermark image which is on behalf of the $i$ row $j$ column pixel gray value. 


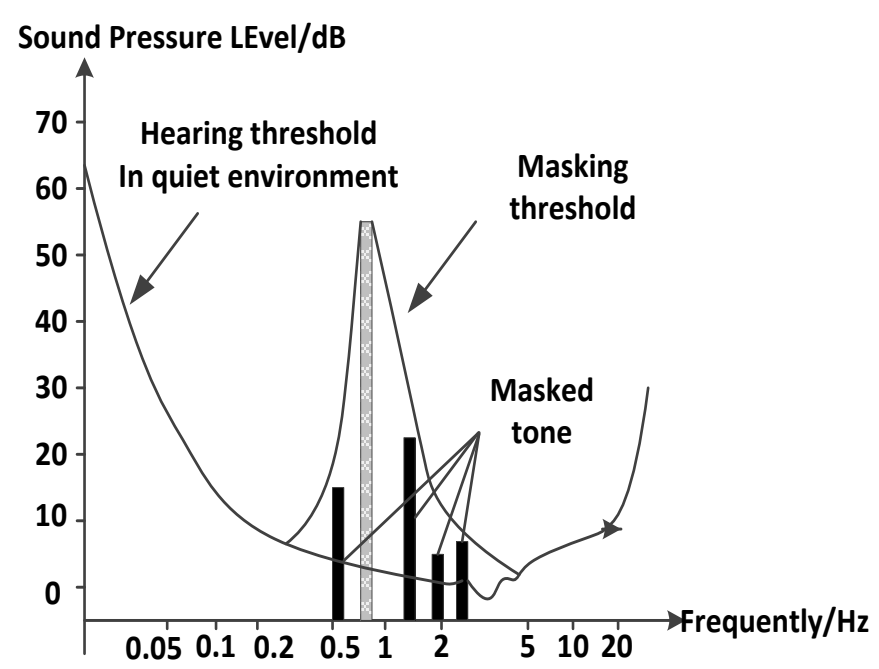

Fig. (2). The mutual masking at the same time between different frequencies of sound.

$w^{\prime}(i, j), 1 \leq i \leq a, 1 \leq j \leq b$ is two-dimensional image scrambling to get the anti-two-dimensional watermark

Robustness is an important standard to measure the robustness of watermarking system, more robust watermark against attacks that ability is stronger. The robustness of the level is divided into the following categories: zero (no beast stick), junior, senior, intermediate, advanced, senior and senior.

The watermark information processing module including image watermark standardization, Arnold scrambling transformation and dimension reduction, the watermark embedding module includes the embedding position and embedding. Watermark embedding module, the original audio signal processing module and detection module includes algorithm quantization watermarking coefficient and self synchronization algorithm, attack module is responsible for the attack on the mixed audio signal simulation. The watermark bits are extracted from the image, need to detect restructure get audio information detection, processing and encryption process is the opposite, first through the Arnold transform of the detected images watermarking information according to the reduction of key by changing the key.

When a certain frequency sound signal a rather high strength, will produce a masking threshold curve on both sides of the A, if there are other sound intensity under this curve, even stronger than the auditory threshold and quiet environment, but the ears of the people that are still could not detect. Masking effect can be further divided into temporal masking and simultaneous masking, temporal masking is refers to between the different time sound mutual masking, is by the human ear to time-resolved decision mechanism, simultaneous masking refers to the mutual masking at the same time between different frequencies of sound, shown in Fig. (1) is the simultaneous masking, simultaneous masking is due the human ear to the voice of the determined frequency resolution mechanism.

Audio signal is divided into meal, every meal of embedding a watermark, audio frame length is determined by the length of the watermark signal, has certain requirements of the original audio signal, the original audio signal to have redundant information embedding watermark enough. After towel continue further subdivides to each PA data, each segment is called a section, each section contains the calculation of audio signal energy, embedding position is determined by the signal energy.

\section{EXPERIMENTAL PERFORMANCE ANALYSES}

Experiment Matlab 7.0 is the platform. because MATLAB has a significant advantage in the treatment of the audio, and after the introduction of the support vector machine, which makes the system development more simple. Moreover, the data display of results, as well as the chart display also has certain advantages, the system supports the use of wavelet transform digital watermarking algorithm of audio watermarking algorithm based on support vector machine for audio file add watermark and extracting watermark function based on.

The audio sample length is $1 \mathrm{~s}$ and the sampling rate are $44.1 \mathrm{kHz}$. The "bird" music is 16bit resolution. Original audio signal and watermarked audio signal are as shown in Fig. (3) and Fig. (5). You can find two audio signals are almost no difference. The hearing can not perceive the subjective existence of watermark when the watermarked audio signals are played.

As shown in Fig. (2) When a certain frequency sound signal a rather high strength, will produce a masking threshold curve on both sides of the A, if there are other sound intensity under this curve, even stronger than the auditory threshold and quiet environment, but the ears of the people that are still could not detect [14]. Masking effect can be further divided into temporal masking and simultaneous masking, temporal masking is refers to between the different time sound mutual masking, is by the human ear to timeresolved decision mechanism, simultaneous masking refers to the mutual masking at the same time between different frequencies of sound, shown in Fig. (1) is the simultaneous masking, simultaneous masking is due the human ear to the voice of the determined frequency resolution mechanism. 


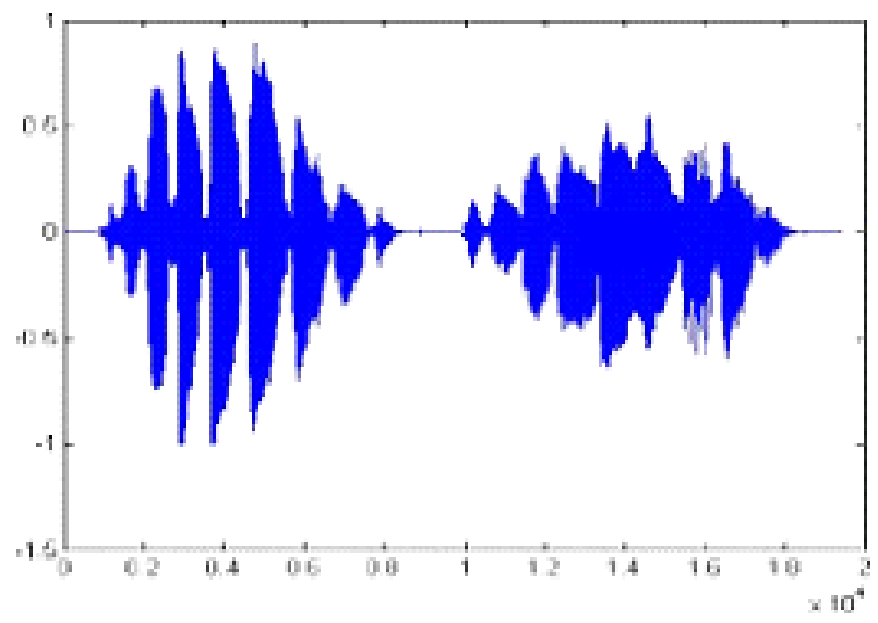

Fig. (3). Original audio signal.

Audio signal intensity as masking tone, weak energy and strong signal masking cannot be signal ear perceive be masking tone. Masking tone in the former, masked tone in the situation called backward masking or forward masking, masked tone in the former, masking tone in the situation referred to as forward masking or backward masking, forward masking, masking is usually exist at the same time, backward masking generally disappeared in 50-200ms masking tone, forward masking in general masking tone appeared before the 5 20ms masking can hide more information. To analyze the digital audio watermarking principle from the angle of signal processing, the process of embedding digital audio watermarking signal is seen as the superposition of weak signal in strong signal, according to the characteristics of human auditory system, as long as the guarantee that the added signal under the human auditory system threshold or in audio signals have a stronger voice can cover up to add sound, the human ear can not feel the signal changes, or feel very small changes. In order to achieve the purpose of embedding watermark will carry on the adjustment to the original audio signal, but considering the time and frequency characteristics of the audio signals, this adjustment is little impact on human hearing it [15-18].

In order to verify the original from an objective point of watermarked audio signal and audio signal can not be aware of, this algorithm uses signal to noise ratio (SNR) of the watermarked audio signal for quantitative evaluation. The higher SNR is, the better is listened. Here we use the quantization factor $\mathrm{Q}=0.01$, the resulting $\mathrm{SNR}=32.7238$. In order to verify the extracted watermark and original watermark similarity, the algorithm uses a normalized correlation coefficient between the two NCs to quantitatively measure the difference. $\mathrm{w}$ and $\mathrm{w}^{\prime}$ are the original watermark and the watermark detection. $\mathrm{NC}\left(\mathrm{w}, \mathrm{w}^{\prime}\right)$ is closer to 1 , and $\mathrm{w}$ and $\mathrm{w}^{\prime}$ are the higher correlation degree. $\mathrm{NC}\left(\mathrm{w}, \mathrm{w}^{\prime}\right)$ is closer to 0 , and $\mathrm{w}$ and $\mathrm{w}^{\prime}$ are the lower correlation degree. Watermark extraction algorithm is based on watermark $\mathrm{NC}=1$.

To further test the robustness of the algorithm, respectively, two different audio signals have a variety of attacks. And similar SNR, with the literature [5] and traditional haar wavelet transform are compared. Fig. (5) is watermarked "bird" music (see Fig. 5) for various attacks on the water- mark image extracted. Table $\mathbf{1}$ is the two audio algorithm and literature used in the watermark embedding and extraction algorithms of $\mathrm{NC}$ values. Table $\mathbf{2}$ is the two audio used haar wavelet-based algorithm and the algorithm to extract the watermark embedding and the $\mathrm{NC}$ value. Attack procedure is as follows:

Table 1. Some common attacks with 2 watermarking audio signals (1 B approximation, 2 NUB wavelet).

\begin{tabular}{|c|c|c|}
\hline Attack & $\begin{array}{c}\text { Bird Music 1 } \\
\text { Q=0.0197snr=30.0754 }\end{array}$ & $\begin{array}{c}\text { Bird Music 2 } \\
\mathbf{Q = 0 . 0 0 3 s n r = 3 2 . 0 8 9 2}\end{array}$ \\
\hline \hline Not attack & 1 & 1 \\
\hline Butter filter & 0.9530 & 0.9562 \\
\hline Gaussian white noise & 1 & 1 \\
\hline Re-quantization & 1 & 1 \\
\hline re-sampling. & 0.9960 & 0.9960 \\
\hline Shear 10\% & 0.9878 & 0.9958 \\
\hline
\end{tabular}

Table 2. Some common attacks with 2 watermarking audio signals (1 $\mathrm{B}$ approximation, 3 haar wavelet).

\begin{tabular}{|c|c|c|}
\hline Attack & $\begin{array}{c}\text { Bird Music 1 } \\
\mathbf{Q = 0 . 0 1 9 7} \mathbf{s n r}=\mathbf{3 2 . 0 7 5 4}\end{array}$ & $\begin{array}{c}\text { Bird Music 2 } \\
\mathbf{Q = 0 . 0 0 3} \mathbf{s n r}=\mathbf{3 2 . 0 7 5 4}\end{array}$ \\
\hline \hline Not attack & 1 & 1 \\
\hline Butter filter & 0.9530 & 0.9575 \\
\hline $\begin{array}{c}\text { Gaussian white } \\
\text { noise }\end{array}$ & 1 & 1 \\
\hline Re-quantization & 1 & 0.9960 \\
\hline re-sampling & 0.9960 & 0.9946 \\
\hline Shear 10\% & 0.9878 & \\
\hline
\end{tabular}




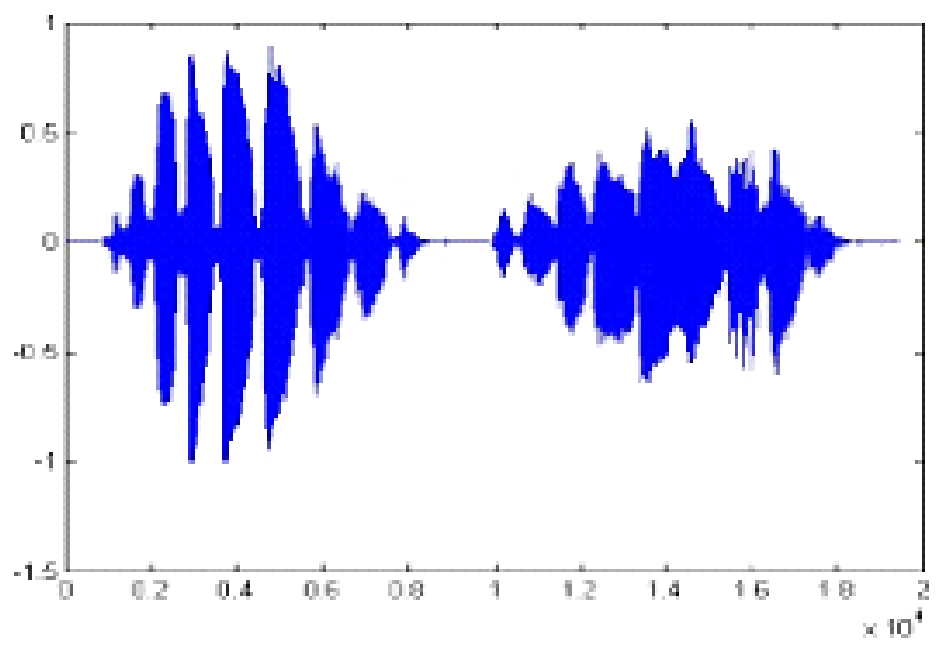

Fig. (4). Audio signal after embedding watermark.

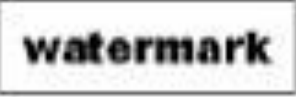

(1) Origina

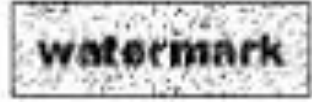

(2) Low-pass filter

\section{watermark}

(3) Gaussian white noise

\section{watermark}

(4) Resampling

\section{watermark}

(5) Re-quantization

\section{watermark}

(6) Shear.

Fig. (5). 4 watermark images extracted after some attacks to audio shown in Fig. (5).

(1) Low-pass filter: Filter order is 6 using cut-off frequency of $22.05 \mathrm{kHz}$. Butterworth low-pass filter filters the audio signal containing the watermark filter, and experimental result is shown in Fig. (5).

(2) Superimposed noise: Watermark for audio signals is superimposed with mean 0 and standard deviation of 0.01 Gaussian white noise, and the experimental result is shown in Fig. (5).

(3) Re-sampling: The original audio watermark signals are up-sampling to $44.1 \mathrm{kHz}$ and down-sampling to $22.05 \mathrm{kHz}$, and the experimental result is shown in Fig. (4).

(4) Re-quantization: The first step contains the watermark from the original 16bit audio signal to quantify the 8bit and then quantifies the 16bit. The experimental result is shown in Fig. (5).

(5) Shear: Audio signals containing random watermark are cut off the original $10 \%$ reduction, and the experimental result is shown in Fig. (5).

(6) The existing audio watermarking method against the problem of poor synchronization attacks, proposes an adaptive synchronous audio watermarking method.

The method makes full use of the characteristics of audio signal, audio signal is divided into frames segmented, choose one of two watermark embedding, watermark embedding is realized through the expansion or reduction of high frequency components of wavelet transform of energy, do not need additional embedded synchronization information, can resist the same step attack, has certain use value [19].

The digital audio watermarking technique because of its wide application has been a hot research aspects of digital watermarking, [20] this paper summarizes the digital audio watermarking algorithms and related techniques, and puts forward the current problems, in order to overcome the algorithm itself in the robustness and the watermark can not be contradiction between perception of both, study the selection of the embedding position, resist attack algorithm, puts forward two kinds of digital audio watermarking algorithm is proposed; based on a digital audio watermarking algorithm based on wavelet transform DWT lower, benefit.

Using the embedding position and relationship to embed digital signal, a digital signal by the quantitative method is embedded into the wavelet coefficients of two adjacent, the experimental results prove that the algorithm has strong robustness, especially robust smoothing attack.

\section{CONCLUSION}

This paper presents a new B-spline approximation based on digital audio watermarking algorithm using B-spline approximation decomposition. The signal length decomposition can be arbitrary.

We can get (less than the original signal) low-frequency components of any size, and traditional wavelet decomposi- 
tion of each signal length must be a decomposition of a multiple of 2, and you can only get low-frequency component of the original signal $1 / 2^{n}$. Reduce the computational volume compared with the non-uniform B-spline wavelet decomposition, so this algorithm has more flexibility. A large number of simulation experiments show that the algorithm can ensure that the watermark can not listen at the same time and the attack on the common have better robustness. This paper presents an audio watermarking algorithm, which is simple and easy to achieve and greater scope.

\section{CONFLICT OF INTEREST}

The author confirms that this article content has no conflict of interest.

\section{ACKNOWLEDGEMENTS}

Declared none.

\section{REFERENCES}

[1] M. Arnold, and P.G. Baum, "A phase modulation audio watermarking technique", In: 11th International Workshop IH. Lecture Notes in Computer Science, 2009, vol. 5806, pp. 102-116.

[2] W. Li, and F.X. Xue, "Localized audio watermarking technique robust against timescale modification", IEEE Transactions on Multimedia, vol. 8, no. 1, pp. 60-69, 2006

[3] Z. Li, C. Li-min, and Q. Gong-bin, "Self-synchronization adaptive blind audio watermarking", In: Proceedings of 12th International Multi-Media Modelling Conference, vol. 1, pp. 381-384, 2006.

[4] W.N. Lie, and L.C. Chang, "Robust and high-quality time-domain audio watermarking based on low-frequency amplitude modification", IEEE Transactions on Multimedia, vol. 8, no. 1, pp. 46-59, 2006.

[5] M. Mansour, and A. Tewfik, "Data embedding in audio using timescale modification", IEEE Transactions on Speech Audio Process, vol. 13, no. 3, pp. 432-440, 2005.
[6] J. Clark, "Some Properties of B-spline", In: Second USA-JAPAN Computer Conference Proceedings, 1975, pp:542-545.

[7] F. Taga, "Smart Music algorithm for DOA estimation", Electronics Letters, vol.33, no.3, pp.190-191, 1997.

[8] W. Sun, J.L. Bai, and K. Wang, "Novel method of ordinal bearing estimation for more sources based on obique projector", Journal of Systems Engineering and Electronics, vol.20, No.3, pp.445-449, 2009 ,

[9] M. Naor, R. Ostrovsky, R. Venkatesan, and M. Yung, "Perfect zero-knowledge arguments for NP using anyone-way permutation", Journal of Cryptology, vol. 11, no. 2, pp. 87-108, 1998.

[10] I. Haitner, O. Horvitz, J. Katz, C. Y. Koo, R. Morselli, and R. Shaltiel, "Reducing Complexity Assumptions for StatisticallyHiding Commitment", In: Proceedings of Advances in Cryptology - EUROCRYPT, 2005, pp. 58-77.

[11] I.Haitner, and O. Reingold, "Statistically-Hiding Commitment from Any One-Way Function”, In: Proceedings of the 39th ACM Symposium on Theory of Computing (STOC 2007), pp. 1-10.

[12] M. Nguyen, S. J. Ong, and S. Vadhan. "Statistical Zero-Knowledge Arguments for NP from Any One-Way Function", In: Proc. 2006 , pp. 66-76.

[13] M. Nguyen, and S. Vadhan, "Zero-Knowledge with Efcient Provers", In: Proceedings of the 38th ACM Symposium on Theory of Computing (STOC 2006), pp. 287-295.

[14] D. Catalano, and I. Visconti, "Hybrid commitments and their applications to zero-knowledge proof systems", Theoretical Computer Science, vol. 37, pp. 229-260, 2007.

[15] O. Goldreich, Foundations of Cryptography (Basic Tools), Cambridge University Press, Cambridge, 2001.

[16] R. Pass, Alternative Variants of Zero-Knowledge Proofs, Licentiate Thesis, Stockholm, Sweden 2004.

[17] C.M. Tang, D.Y. Pei, and Z.A. Yao, "Efficient Zaps and Signatures of Knowledges", In: Proceeding of IEEE International Conference on Computational Intelligence and Security(CIS'2007), 2007, pp. 637-641.

[18] K. Li, H. Chen, Y. Chen, D.W. Clark, P. Cook, S. Damianakis, G. Essl, A. Finkelstein, T. Funkhouser, and T. Housel, "Building and using a scalable display wall system", IEEE Computer Graphics and Applications, vol. 20, no. 4, pp. 29-37, 2000

[19] R. Yang, D. Gotz, J. Hensley, H. Towles, and M.S. Brown "Pixelflex: A Reconfigurable Multi-Projector Display System”, In: Proceedings of the conference on Visualization'01, 2001, pp. 167174.

[20] B. Wei, C. Silva, E. Koutsofios, S. Krishnan, and S. North, "Visualization research with large displays", IEEE Computer Graphics and Applications, vol. 20, no. 4, pp. 50-54, 2000.

Received: September 22, 2014

(C) Ping Ren; Licensee Bentham Open.

This is an open access article licensed under the terms of the Creative Commons Attribution Non-Commercial License (http://creativecommons.org/licenses/by-nc/3.0/) which permits unrestricted, non-commercial use, distribution and reproduction in any medium, provided the work is properly cited. 